[To be published in the Proceedings of the Society of Photo-optical Instrumentation Englneers Meeting, to be held September 14-26, 1986,
Cambridge, MA]

CON F-8609127--4

DE86 015413

\title{
FUNDAMENTAL ASPECTS OF PULSED-LASER IRRADIATION OF SEMICONDUCTORS
}

\author{
G. E. Jellison, Jr., D. H. Lowndes, and R. F. Wood
}

\section{DISCLAIMER}

This report was prepared as an account of work sponsored by an agency of the United States Government. Neither the United States Government nor any agency thereof, nor any of their employees, makes any warranty, express or implied, or assumes any legal liability or responsibility for the accuracy, completeness, or usefulness of any information, apparatus, product, or process disclosed, or represents that its use would not infringe privately owned rights. Reference herein to any specific commercial product, process, or service by trade name, trademark, manufacturer, or otherwise does not necessarily constitute or imply its endorsement, recommendation, or favoring by the United States Government or any agency thereof. The views and opinions of authors expressed herein do not necessarily state or reflect those of the United States Government or any agency thereof.

\author{
SOLID STATE DIVISION \\ OAK RIDGE NATIONAL LABORATORY \\ Operated by \\ MARTIN MARIETTA ENERGY SYSTEMS, INC. \\ under \\ Contract No. DE-AC05-840R.21400 \\ for the \\ U.S. DEPARTMENT OF ENERGY \\ OAK RIDGE, TENNESSEE 37831
}

August 1986 


\title{
Fundamental appects of pulsed-laser irradiation of semiconductora
}

G. E. Jellison, Jr., D. H. Lowndes, and R. F. Wood

Solid State Division, Oak Ridge National Laboratory

Oak Ridge, Tennessee 37831

\begin{abstract}
Fundamental aspects of pulsed laser melting and solidification of crystalline siljcon ano germanium are reviewed. The discussion concentrates on time-resolved experiments performed with nanosecond pulsed lasers, although some picosecond and femtosecond experiments are also considered. The creation of amorphous material from crystalline material induced by ultrarapid melting and resolidification using either nanosecond or picosecond lasers is surveyed and the inverse process of rerrystallization of a-Si by explosive crystallization is described. Finally, melting model calculations, which have proven to give a very accurate description of the pulsed laser irradiation process, are discussed.
\end{abstract}

\section{Introduction}

A wide range of effects resulting from the irradiation of semiconductors with pulsed lasers having $10-50 \mathrm{~ns}$ pulse widths have been the subject of extensive study for the last 8-10 years and the nature of the underlying process is well established: If a light pulse of sufficient energy density is incident upon the surface of a semiconductor, the nearsurface region melts.1 The melt front first moves very rapidly into the material, pauses, and then a solidifying front returns less rapidly to the surface. If the surface has been amorphized by ion implanta.ion or some other technique, and the melt front penetrates beyond the amorphous-crystalline (a-c) interface, then single crystalline epitaxial iegrowth will generally occur, provided the regrowth velocity is less than $\sim 5 \mathrm{~m} / \mathrm{s}$. Transmission Electron Microscopy (TEM) shows that the material regrown in this manner has far fewer dislocation loops than furnace-annealed ion implanted material. The characteristics of the melt, including the melt duration, the time of the onset of melting and the maximum melt depth penetration depend in a complicated fashion on the materials properties and on the parameters of the laser pulse, including its wavelength, pulse duration, shape, and energy density.

Pulsed excimer lasers have certain characteristics that have allowed them recently to play a particularly important role in both the fundanental and applied studies of pulsedlaser irradiation of semiconductors:

(1) Compared with solid state lasers, excimer lasers are generally very incoherent ligi:t sources, because several thousand different transverse and longitudinal modes can lase simutaneously. The principal advantage of this for fundamental studies is that diffraction effects are minimized, and the transverse beam homogeneity is improved, in comparison with solid state lasers. This improved homogeneity is achieved without the added complication of diffuser plates and/or light pipes, which have been used to improve the transverse homogeneity of solid state lasers.

(2) Because excimer lasers put out ultraviolet light, the light is essentially absorbed in the first $10-20 \mathrm{~nm}$ of most semiconductors. From the point of view of most heat flow calculations on the ns time scale, this is effectively the surface of the sample, and the absorption coefficent no longer is a critical parameter in the calculations. of course, the reflectivity is still very important in determining fow much light energy is coupled into the near-surface region. Since the reflectivity of semiconductors is not well known in the UV, particularly at higher temperatures and in the liquid phase, this creates some uncertainty in the results of model calculations.

(3) The pulse-to-pulse reproducibility of the pulse shape and energy density of excimer lasers is generally much better than that of solid state lasers. This makes the comparison of results from different but nominally similar pulses mich easier. In this case, signal averaging become: a reasonable experimental technique, particularly if the pulseto-pulse differences in the pulse energy are recorded, and energy bin signal averaging is performed.

(4) Excimer lasers are generally more robust and reliable over long time periods than solid state lasers. This is particularly important for the experimenter who wants to 
concentrate on the fundamental aspects of the laser annealing process without worrying too much about the laser itself.

In this paper, we highlight some of the experiments and calcuiations performed in recent studies of pulsed-laser irradiation of semiconductors. Although many of these experiments were performed using pulsed excimer lasers, as the heating (or pump) laser, we also include results obtained with nanosecond ruby and Nd:YAG lasers, as well as some results from picosecond and Eemtosecond lasers. We focus on experiments performed on silicon, but some results for germanium will also be discussed.

\section{Experiments}

\section{Time-resolved optical measurements}

Nanosecond experiments. Time-resolved reflectivity (TRR) measurements with nanosecond resolution were the first performed to examine the nature of the phase change induced by pulsed-laser irradiation of semiconductors. These measurements are carried out by focusing a low-power $c w$ probe laser on the spot irradiated by the heating laser, and detecting the reflected light from the probe laser with a suitable detector, such as an avalanche photodiode (APD) or a p-i-n diode. The first TRR experiments, on silicon irradiated with a pulsed ruby laser, were performed by Sooy et al.,2 who found that the reflectivity underwent a large increase which lasted for several tens of nanoseconds after laser irradiation, and was attributed to a phase change to a metallic state. Later TRk experiments by Auston et al. ${ }^{3}$ attributed the increase in reflectivity to melting of the semiconductor surface, since many liquid semiconductors are metallic and therefore have a higher reflectivity than the hot solid for probe wavelengths longer than the direct band gap.

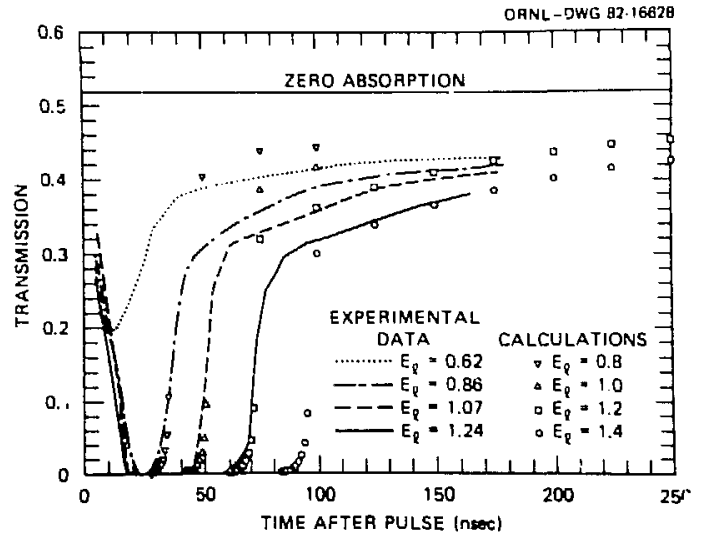

Figure 1. Time-dependent transmission of a $1152 \mathrm{~nm}$ focused probe laser beam through crystalline silicon during and after pulsed ruby laser irradiation with 15 ns pulse width (full width at half maximum). The lines show the experimental data, while the data points indicate the transmission calculated from melting model calcularions. The energy densities $\left(E_{1}\right)$ are given in units of $\mathrm{J} / \mathrm{cm}^{2}$, and the horizontal line at 0.52 indicates the transmission for no internal absorption (from Ref. 4).

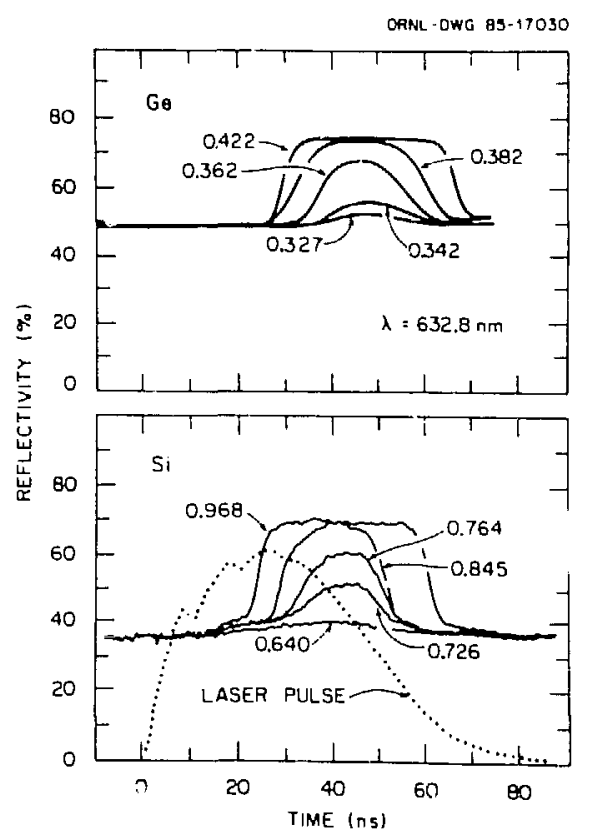

Figure 2. Time-resolved reflectivity of $\mathrm{Ge}$ (top panel) and $\mathrm{Si}$ (bottor panel) at $633 \mathrm{~nm}$ during pulsed KrF laser irradiation. The time profile of the laser pulse is shown in the botton panel, for which the axis is in arbitrary units. The numbers in the figure refer to the $\mathrm{Kr} F$ laser energy density in $\mathrm{J} / \mathrm{cm}^{2}$ (from Ref, 6).

To examine further the question of whether silicon actually melts during pulsed ruby laser irradiation, Lowndes et al.4 carried out a series of TRR and time-resolved transmission (TRT) experiments. The TRT experiments were performed by passing a cw 1152 
no HeNe laser beam through a sample of crystalline silicon, which is nearly transparent to light of this wavelength at room temperature. Therefore, the radiation from the cw laser could be detected by an APD placed directly behind the sample, the only attenuation in the intensity being due to reflection losses at the front and back surfaces of the sample.

The resulting transmission vs time is shown in Fig. 1 for several different pulsed ruby laser energy densities. It was found that the intensity of the transmitted beam first decreased and then went to zero, stayed at zero for a time dependent upon the laser energy density, and then increased to a non-zero value, but less than the intensity observed before the experiment. Well after the laser pulse was over, the transmitted intensity was observed to return slowly to the pre-irradiation value. Tue decrease in transmitted intensity before zero transuission, and the slow return of the transmitted intensity to the pre-irradiation value, can be explained largely by heating of the silicon nearsurface region, since the absorption coefficient of silicon at 1152 nm increases dranatically with increasing temperature.5 During the time that the near-surface region of the silicon sample is in the high reflectivity (molten) phase, the silicon sample is opaque to $1152 \mathrm{~nm}$ light, further indicating thdt the high reflectivity phase corresponds to a metallic phase.

More recently, Jellison et al.o have conducted a series of TRR measurements during pulsed KrF laser irradiation of $\mathrm{Si}$ and $\vec{G}$. A typical series of TRR traces lising a 632.8 no HeNe probe laser are shown in Fig. 2. Several time domains can be identified from the TRR trace:

(1) As soon as the laser pulse turns on, the sample surface begins to heat up. The reflectivity changes, increasing monotonically for Si, but increasing and then decreasing for Ge. These observations can be understood completely from the temperature dependences of the optical properties of $\mathrm{Si}$ and $\mathrm{Ge}$, and are completely consistent with the sample surfaces heating up before melting occurs.

(2) Once the surface temperature [inferred Eron $R(T)$ ] reaches the melting point of the material, surface melting begins. The melting process is discussed in more detall below.

(3) If the energy density is sufficiently high, the entire front surface region uelts to a depth of at least several optical skin depths. This is shown in Fig. 2 for the highest energy density in each panel by the reflectivity increasing to a constant value. The total surface melt duration time, and the time of the onset of melting, depend critically upon the energy density of the heating laser (shown in Fig. 3 ).

(4) Once the melt front is created, it propagates into the material, pauses, and then a solidifying front returns to the front surface. When the solidifying front is within $\sim 20$ no of the front surface, the reflectivity begins to decrease, reaching the value for hot material at the melting point when the last remaining molten material disappears.

Time-resolved ellipsometry. Recently, Jellison and Lowndes ${ }^{7}$ performed time-resolved elTipsometry (TRE) measurements during pulsed KrF laser irradiation of silicon using a $632.8 \mathrm{na}$ probe laser. Ellipsometric measirements utilize a large angle of incidence and allows one to measure the ellipsometric angles $\psi$ and $\Delta$, as well as the polarized reflectivities $R_{S}$ and $R_{R}$ (the reflectivities for light polarized perpendicular and parallel to the plane of incidence, respectively). From these measurements, the optical functions of liquid silicon were determined to be $n=3.8$ and $k=5.2$ at $632.8 \mathrm{~nm}$. In addition, the effective surface refractive index and extinction coefficient can be measured and related to the temperature through the results of temperature-dependent optical function measurements of silicon. The temperatures determined in this manner indicated that the surface temperature reached $\sim 1400^{\circ} \mathrm{C}$ before the surface melts.

However, upcn closer examination of the TRE data, it wals found that a simple melt-in model, in which a well-defined planar interface between the liquid and hot solid exists, and progresses uniformly into the sample as more laser energy is absorbed, is not possible.7 Using such a model the thickness of the liquid layer predicted from the $\psi$ measurements is vastly different from the thickness of the liquid layer determined from the $\Delta$ measurements. A more realistic model is one in which the near-surface region is assumed to consist of a layer in which liquid and solid coexist as melting begins. If the near-surface region is modeled as an effective medium, $\theta$ two free parameters (the thickness of the effective medium $D$ and the fraction of solid $F_{S}$ ) can be determined from the TRE parameters $\psi$ and $\Delta$ as functions of time. The results are shown in Fig. 4 (a) for silicon irradiated with $0.8 \mathrm{~J} / \mathrm{cm}^{2}$, just above the melting threshold $\left(0.65 \mathrm{~J} / \mathrm{cm}^{2}\right)$. With the values of $D$ and $F_{s}$ determined, the reflectivities $R_{s}$ and $R_{p}$ can be calculated and compared with experimental values determined from the TRE experiments. These comparisons are shown in Fig. 4(b), and show that this model is internally consistent. 

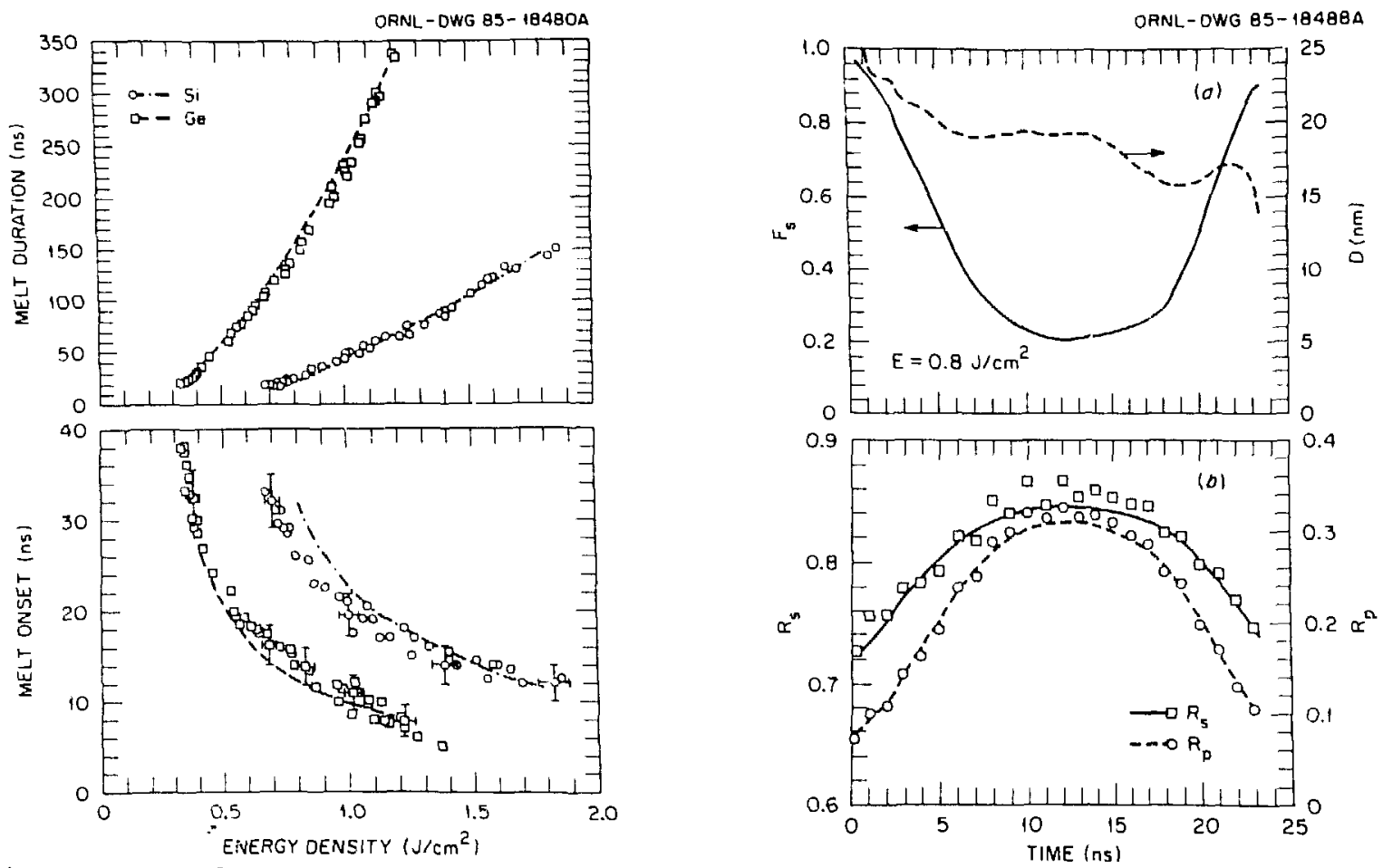

Figure 3. Surface melt duration (top) and time of the onset of melting (bottom, rela-

Figure 4. a) Effective medium thickness $D$ and fraction of solid $F_{s}$ calculated from $\phi$ and $\Delta$ using the effective medium theory, for $\mathrm{Si}$ irradiated at $0.8 \mathrm{~J} / \mathrm{cm}^{2}$. b) The reflectivities for light polarized parallel $\left(R_{p}\right)$ and perpendicular $\left(R_{s}\right)$ to the plane of incidence; the lines represent $R_{s}$ and $R_{p}$ calculated from the effective mediup thickness $D$ and the fraction of solid $\mathbf{F}_{\mathbf{g}}$ given in a) above, while the points represent actual experimental data. Time $=0$ corresponds to the onset of melting (from Ref. 6).

For Si irradiated with $0.8 \mathrm{~J} / \mathrm{cm}^{2}, F i g .4(a)$ shows that $F_{s}$ decreases continuously to a value of 0.2 at 12 ns after the onset of melting, when it starts to increase again to a value of 1 at 24-25 ns after the onset of melting. During this time, the thickness of the effective nedium layer remains relatively constant at $20 \mathrm{~nm}$. For a more intense laser pulse ( $1.4 \mathrm{~J} / \mathrm{cm}^{2}$, not shown), the fraction of solid decreases continuously from 1 to zero, 7 ns being needed to completely melt the near-surface region.

The picture suggested by the effective medium model is one in which liquefaction of the near-surface region occurs as a bulk process, with liquid inclusions forming within the top $20 \mathrm{~nm}$ and then growing as more laser energy is absorbed. The inhomogeneous melting apparently revealed by these experiments could be due to one or more of the following; (1) inhomogeneities in the spatial distribution of the laser radiation; (2) microscopic imperfections of the sample surface; and/or (3) the phase nucleation process itself.

Eemtosecond experiments. One of the central assumptions In analyzing the nanosecond experiments using a thermal model is that the transfer of energy from the laser-excited electron-hole pairs to the crystal lattice takes place in a time that is comparabie to or less than the nanosecond or longer duration pulses used for laser irradiation of semiconductors.

A detailed understanding of this thermalization process actually requires treating two processes that occur simultaneously: (1) The dynamics of a hot electron-hole plasma whose density may be varying rapidly in time and (2) the process of energy rransfer from these excited carriers to the lattice via the electron-phonon interaction (see Refs. 1, 9, and 
10 Eor a more in-depth discussion). Electron-hole pairs are produced by the absorption of laser light in direct or indirect transitions across the energy bandgap; free-carrier absorption also increases with increasing carrier density. The hot carriers resulting from both processes then thermalize, both with other carriers and with the lattice. A common carrier temperature for electrons and holes is expected to be established it $<10$ femtoseconds as a result of electron-electron and electron-plasmon collisions; concurrent with these intra-carrier equilibration processes, hot carriers can transfer energy to the lattice via electron-phonon scattering, in a time expected to be sl picosecond.9 A series of electron-phonon interactions results in electrons trickling down to the bottom of the conduction band (or holes to the cop of the valence band) at which point the remaining band-gap excitation can be lost by electron-hole recombination. For the timescale and carrier densities of interest in laser processing the predominant electron-hole recombination process is the Auger process, in which the bandgap racombination energy of an electron-hole pair is given to a third carrier (either an electron or a hole). Although che Auger process does not reduce the net energy of the carrier system, it does reduce the carrier density and creates hot carriers whose excess kinetic energy can be given up to the lattice via further electron-phonen scattering.

Shank and collaborators 11 have used 90 Eemtosecond ( 1 fentosecond a 10-15 sec) optical pulses to study the process of energy transfer from a laser-excited plasma co the silicon crystal lattice and to observe the melting phase transition in silicon with subpicosecond resolution. In Shank's experiments, melting was observed well after, rather than during the excitation pulse, so that the reflectivity signature of plasma formation was observed unambiguously prior to melting. The time-resolved reflectivity measurements were carried out by the excite-and-probe technique, where the sample is irradiated by two laser pulses: The excite pulse of relatively high energy, and the probe pulse usually split off from che excite beam, delayed in time, of lower power, and possibly frequency-shifted. The reflectivity is th* recorded by a solid state detector connected to a sample-and-hold circuit.

If a plasma is formed, then the reflectivity depends upon the density of the plasma from the Drude expression for the refractive index:

$$
n_{p}=n_{0} \sqrt{\left(1-\omega^{2} p / \omega^{2}\right)}
$$

where $n_{O}$ is the high frequency refractive index ( $n_{O}=3.5$ for $S i$ ) and $\omega^{2} p^{2}=4 \pi \mathrm{Ne}^{2} / \mathrm{m}^{*} \mathrm{E}_{\mathrm{s}}$ is the square of the plasma frequency. An increase or decrease of the reflectivity will be observed with increasing carrier density $N$, depending on the Erequency of the probe light $\omega^{2}$. The quantity $\omega^{\star}$ is the carrier effective mass, and $\varepsilon=n_{0}{ }^{2}$.

The measurements of Shank and collaboratorsll demonstrate a loss of erystalline order on the (111) silicon surface that is consistent with melting occurring on a time scale of substantially less than one picosecond. Their reflectivity measurements show that the melting process is apparently initiated at the surface, but is preceeded by the formation of a dense $\left(>10^{21} / \mathrm{cm}^{3}\right)$ electron-hole plasia in the near-surface region.

\section{Iine-resolved $x-r a y$ measurements}

Time-resolved $x$-ray diffraction provides a direct means for probing the near-surface structure of a crystal lattice during pulsed laser irradiation. ${ }^{2}$ Using this method, information about the temperature can in principle be obtained from the thermal expansion, and the onset of melting and recrystallization can be monitored through the disappearance. and reappearance of Bragg diffracted intensity from this region. Nanosecond resolution was obtained by using syncrotron $x-r a y$ probing pulses of $1.5 \mathrm{~A}$ wavelength and 0.15 ns duration.

Figure 5 shows the measured $x$-ray scattering of Larson et a1.12 at the (111) reflection of crystalline silicon during the melt induced by $1.5 \mathrm{~J} / \mathrm{cm}^{2}$ ruby laser pulses of 15 ns pulse duration. The data listed 20 ns were taken just after the end of the laser pulse, while the data for 55 ns were taken while the near-surface region was entirely molten. Through a proper analysis of the data in Fig. 5, one can determine the strain as a function of depth in the solid; if there is a liquid layer on the surface, then the measurement begins at the liquid-solid interface. If the temperature-strain relationship is known accurately under the conditions of the experiment, it then is possible to deduce the temperature of the hot solid as a function of depth, shown in Fig. 6 .

As can be seen from Fig. 6, the temperature of the liquid-solid interface (Depth=0 on this plot, for $t_{\mathrm{L}}=20 \mathrm{~ns}$ and $\left.55 \mathrm{~ns}\right)$ reaches the melting point of silicun (1410 $\left.\mathrm{C}\right)$, with little or no interfacial overheating or undercooling. For times weil after the end of near-surface melting $\left(t_{\mathrm{L}}=155 \mathrm{~ns}\right)$, the maximum temperature at the surface is $1050^{\circ} \mathrm{C}$, while 
the temperature gradient going into the material has decreased; this indicates surface cooling and heat flow into the bulk of the sample, as would be expected following resolidification of a melted surface layer.

Ore characteristic of these $x$-ray measurements is that signal averaging is necessary. Since the heating laser used. in the experiments described in Refs. 12 was a ruby laser, an uncertainty enterej the experiments and their interpretation due to pulse-to-pulse variations. To correct these problems, Larson et al.l3 performed these experiments again using an excimer laser, obtaining very similar results. However, the increased accuracy of their experiments resulted in them being able to deduce an overheating and a undercooling during melting and regrowth, respectively, of $110 \pm 30 \mathrm{~K}$ for $\langle 111\rangle \mathrm{Si}$, and $50 \pm 25 \mathrm{~K}$ for $\langle 100\rangle \mathrm{Si}$.

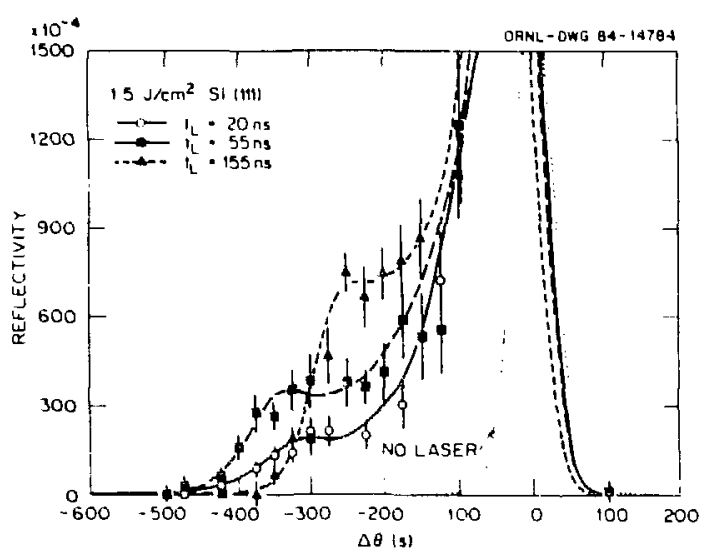

Figure 5. Measured $x$-ray scattering at 20,55 , and 155 ns after the center of a $15-\mathrm{ns}, 1.5 \mathrm{~J} / \mathrm{cm}^{2}$ ruby laser pulse for the $\mathrm{Si}(111)$ reflection (from Ref. 12).

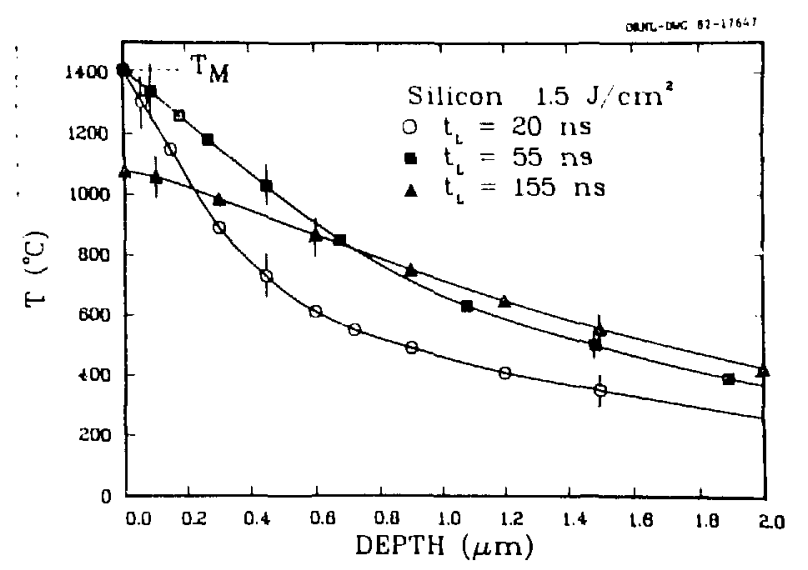

Figure 6. Time-resolved temperature profiles corresponding to the $x-r$ ay measurements in Fig. 5 (from Ref. 12).

\section{Time-resolved electrical conductivity experiments}

The duration of melting and the velocity of the recrystallizing interface produced by pulsed laser irradiation of silicon have been inferred fcom time-resolved reflectivity measurements and from model calculations. However, more direct measurements of the velocity of the liquid-solid interface and of the total depth of molten silicon have been carried out using a time-resolved electrical conductivity technique.14,15

The technique consists of measuring the conductance of a silicon stripl4 or (in later experiments) of a thin silicon-on-sapphire (SOS) filmb during and after uniform irradiation of the sample by 3-30 nanosecond ruby laser pulses, taking advantage of the fact that $\mathrm{Si}$ undergoes a large decrease in electrical resistivity upon melting. Low lifetime Au-doped silicon was used in the first experiments to reduce the photoconductance due to photogenerated-free carriers at long times after the laser pulse. By using a sample with a large length/width ratio (most easily achieved by photolithographic patterning of Sosis) the resistance of the sample can be made much larger than the contact resistance ( $2-3 \Omega$ ), even at the time of maximum melt depth. After the photoconductance has decayed, the sample conductance $G \times\left(w d / l_{p}\right)$, where $w$ and $l$ are the width and the length of the sample, $d$ is the depth of the molten layer, and $\rho$ is the resistivity of molten silicon ( $80 \mu \Omega-c m$ ). Thus, a plot of sample conductance vs time can be scaled to give melt depth vs time, with the velocity of the liquid-solid interface during recrystallization given by the slope of the plotted curve.

For energy densities greater than the energy density required for melting (about 0.9 $\mathrm{J} / \mathrm{cm}^{2}$ for $30 \mathrm{~ns}$ ruby pulses) time-resolved sample conductances (or equivalently, melt depths) can be obtained from the curcent transients; these are shown in Fig. 7 (a). The vertical broken line at 135 nanoseconds shows the approximate duration of photoconductance. Galvin et al. 4 also carried out melting model calculations of the time-dependent conductivity to compare with their experiments, as shown in Fig. 7 (b). If only the conductivity of the liquid layer is included, then the calculated conductance falls below the experimental conductance, but if the conductivity of the solid is included in the calculation, much better agreement with experiment is obtained. 


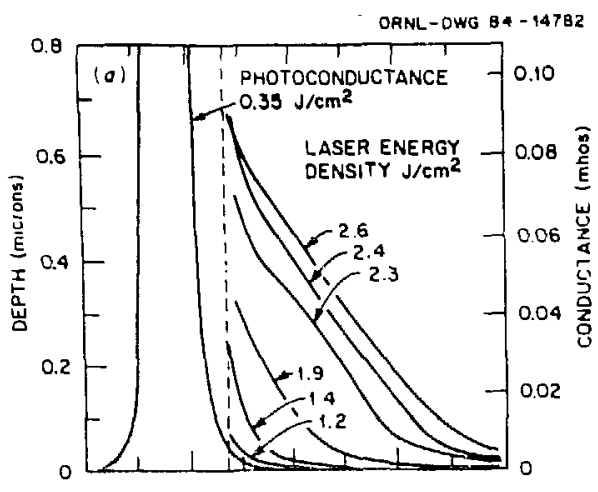

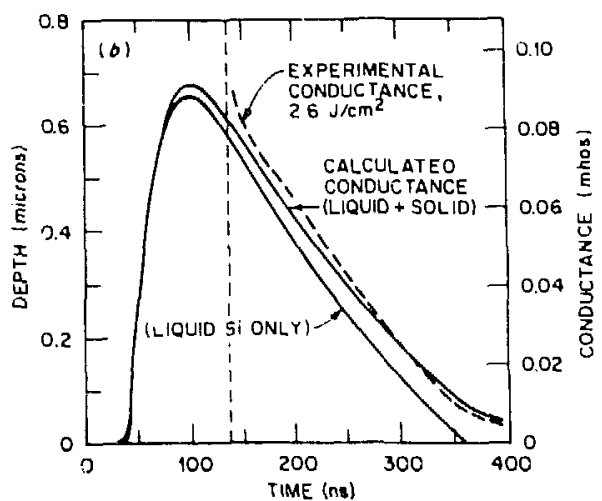

Figure 7. (a) Conductance vs time. The depth scale is derived by assuming the conductance is determined only by the conductivity of the molten silicon. (b) Calculated curves, based on a thermal heat flow model, of the conductance of liquid and near-interface solid $\mathrm{Si}$ (upper curve) and liquid $\mathrm{Si}$ only (lower curve). The dashed line is the experimental curve (from Ref. 14).

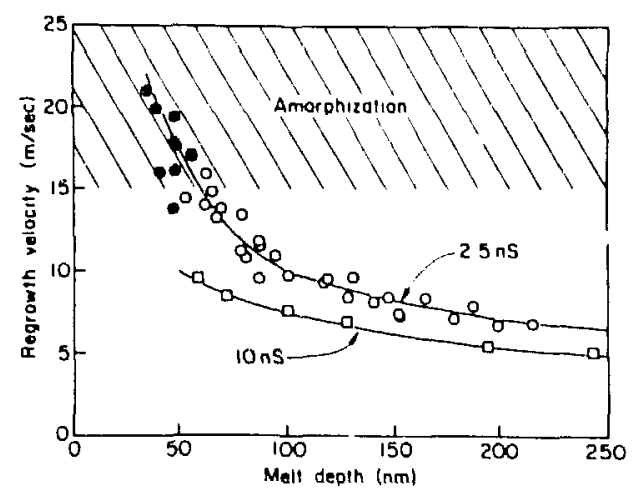

Figure 8. Regrowth velocity vs melt depth for pulsed UV-irradiated SOS. The hatched area and the solid data points represent the regime in which amorphous silicon forms from the melt (from Ref. 15).

\section{Liquid-to-amorphous phase transformation}

It is known that thin layers of amorphous silicon can be formed by ultrarapid solidification from a shallow pool of molten silicon that is produced by pulsed laser irradiation of a crystalline silicon surface. Either nanosecond pulses of ultraviolet light or picosecond pulses of visible and near-infrared light may be used.15,16 The amorphous phase forms as a result of ultrarapid cooling of a very thin molten layer by conduction to the underlying crystalline substrate. The critical influence of cooling rate on amorphous phase formation is illustraced by the fact that the amorphous layer can be formed only if the pulsed laser fluence lies within a narrow energy density "window, ' just above the energy density needed to melt the front surface. Higher fluences result in melting to a sufficient depth that thermal energy stored in the liquid layer prolongs the duration of melting, with cooling and solidification then occurring sufficiently slowly that epitaxial regrowth from the crystalline substrate results. Thus, amorphous phase formation is favored by the use of a pulsed laser source having a short absorption length and short pulse duration. Amorphous layers are formed more easily on the (111) Si surface than on the (100) surface, although the reason for this is not yet ciear.

Thompson et al.15 recently applied the transient cunductance technique to directly measure the velocity of the solidifying melt-solid interface under conditions such that the liquid-to-amorphous transition of silicon occurs. Using a 2.5 nanosecond, 347-nm laser (from a frequency doubled ruby laser), amorphous regrowth was observed only for energy densities between 0.2 and $0.3 \mathrm{~J} / \mathrm{cm}^{2}$, with the maximum depth of a. orphous silicon of $14 \mathrm{~nm}$ being obtained for an energy density of $0.27 \mathrm{~J} / \mathrm{cm}^{2}$. Figure 8 shows the regrowth velocity plotted as a functicn of maximum melt depth. As can be seen from Fig. 8 , amorphization of a (100) SOS surface occurs for regrowth velocities exceeding $15 \mathrm{~m} / \mathrm{s}$. 
Closely related measurements of the melt-solid interface velocity were also carried out by Bucksbaum and Bokor, 17 who used an excimer laser (KrF, $248 \mathrm{~nm}, 15$ picosecond pulse width) to melt silicon, and a delayed $1 . t+\mu$ probe pulse to determine the thickness of the molten silicon Eiln on the picosecond time scale, using both reflectivity and transmission measurements. Profiles of melt depth vs time were obtained for both (100) and (111) crystalline silicon surfaces, for Eluences of $0.03-0.11 \mathrm{~J} / \mathrm{cm}^{2}$, corresponding to maximum melt durations Erom 200 picoseconds to 22 manoseconds, and resulting in a nearly constant solidification velocity of about $25 \mathrm{~m} / \mathrm{s}$ over about $80 \%$ of the duration of amorphous regrowth. Liquid Eilms up to $40-n m$ thick were found to be Eully amorphized upon resolidification.

\section{Amorphous to fine-graized polycrystalline Si phase transformation}

It is also possible to create fine-grained and large-grained polycrystalline Si from amorphous Si. In these experiments, the samples consisted of crystalline Si (wafers or sos) whose surface has been amorphized by ion implantation with Si atoms to a depth of $20-50$ nm. Thompson et al.lB first examined the time-resolved conductivity in conjunction with TRR measurements, where they found that the conductivity measurements indicated the existence of liquid $S i$ at times that the TRR signal did not; they concluded that this indicated the existence of a buried molten layer, that is, a layer of molten si not contiguous with the surface. TEM sturies of these samples revealed that laser-irradiated material consisted of a layered structure: large-grained polycrystalline Si, small-grained polycrystalline Si, aworphous $\mathrm{Si}$, and crystaline $\mathrm{Si}$.

In a later set of experiments, Lowndes et al.19 examined the TRR of amorphized Si during pulsed KrF laser irradiation using two $\mathrm{Cw}$ probe beams (633 and 1152 nm) simultaneously. Because the 1152 nm probe bedm is much less attenuated in amorphous or crystalline si, they found that they could follow the propagation of this buried molten layer as a function of time, and, by recording the maxima and minima of the $1152 \mathrm{~nm}$ TRR, determine the velocity of propagation of the buried molten layer.

Lowndes et al.19 also examined some amorphized Si samples that had additional Cu atoms implanted as a marker. Copper has a very low solid solubility in Si (and a low interface segregation coefficient); therefore, if a buried molten layer is generated, the segregation of $\mathrm{Cu}$ to a point deep inside the anorphized layer should be observed. After pulsed KrF laser irradiation, both Rutherford backscattering and $Z$-contrast STEM measurements were made, indicating that $C u$ was inrzed transported deeper into the amorphized layer by the buried molten layer. The buried layer thickness was determined to be $<15$ nn at the time that it solidified, and the final position of the buried layer lay at the boundary between the fine-grained polycrystalline $S i$ and the amorphous Si.

\section{Melting model calculations}

It was noted above that the time required to convert light energy from a laser pulse to thermal energy is 61 picosecund. Therefore, the energy input by nanosecond lasers can be treated by the usual heat diffusion equation with the laser pulse as a heat source term capable of inducing melting (see Refs. 1 and 20).

The application of this "melting model" to an actual pulsed laser annealing experiment requires knowledge of the temperature dependences of the thermal properties of the material under study. The thermal conductivities of solid $\mathrm{Si}$ and Ge have been measured as a function of temperature by many groups, but particularly accurately by Glassbrenner and Slack.21 However, the thermal conductivities of liquid $\mathrm{Si}$ and Ge have not been accurately measured and, therefore, the Wiedemann-Franz law io sometimes employed to obtain the thermal conductivity of liquid semiconductors. Graphs of the temperature-dependent thermal conductivities are given in Chapter 4 of Ref. 1. The temperature-dependent specific heats of solid Si and Ge can be obtained from the compilation of Hultgren et al.22 The specific heat of liquid Si and $\mathrm{Ge}$ also has not been accurately measured, and it is normally assumed to be equal to that of the solid at the melting temperature.

It is also important to know the optical absorption coefficient a and the normal-incidence reflectivity $R$ as input parameters of the melting model calculations, but this data is not as readily available. For laser annealing of Si with wavelength well below the direct band gap $(3.4 \mathrm{eV}$ at $300 \mathrm{~K})$, the temperature dependence of a and $\mathrm{R}$ for the solid phase are known empirically to be ${ }^{2} 3$

$$
a(\lambda, T)=a_{0}(\lambda) \exp \left(T / T_{0}\right)
$$

and

$$
R(\lambda, T))=R_{0}(\lambda)+a(\lambda) T .
$$




\section{7}

where $\lambda$ is the wavelength of light, $T$ is the temperature, $T_{0}=430^{\circ} \mathrm{C}$ and $a_{0}(\lambda)$ and $R_{0}(\lambda)$ are the values at $\mathrm{T}=0$.

For the case of solid Ge, the data are more complicated, since an important direct band gap is $\sim 2.2 \mathrm{eV}$ at $300 \mathrm{~K}$, which moves down to $\sim 1.6 \mathrm{eV}$ at the melting point of Ge. The data of Vinà et al.24 can be used from 1.5 to $5.5 \mathrm{eV}$, and from room temperature to $\sim 825^{\circ} \mathrm{C}$. For the liquid, a is large $\left(210^{6} / \mathrm{cm}\right)$ and therefore is not an important parameter in the calculations, but $R$ is important. $R$ can be determined approximately by interpolating the data of Shvarev et al.25 for $\mathrm{Si}$ and of Hodgson26 For Ge.

For laser irradiation by ultraviolet lasers, the optical functions of $\mathrm{Si}$ and Ge are not accurately known. The absorption coefficient is known to be large ( $10^{6} / \mathrm{cm}$ ) for both liguid and solid $\mathrm{Si}$ and $\mathrm{Ge}$, and therefore introduces no majcr uncertainty into the melting model calculations. However, the reflectivity does have a significant effect on the results of the melting model calculations. In particular, the calculated melt duration and the calculated time of the onset of melting are very sensitive to the reflectivities assumed for the liquid and the solid, respectively. By varying the reflectivity of the solid in melting model calculations, and comparing the results with experiment, Jellison et al.6 Found that the reflectivity at $248 \mathrm{~nm}$ for solid $\mathrm{Si}$ was $63 \%$ and for solid Ge, $60 \%$. Similarly, by varying the reflertivity assumed for the liquid and comparing the results of the melting model calculations with experimental values of the surface melt duration, they found that the reflectivity at $248 \mathrm{~nm}$ of liquid $\mathrm{Si}$ was $70 \%$ and of liquid Ge, $65 \%$. These measurements and calculations are shown in Fig. 3 as a function of the KiF laser energy density.
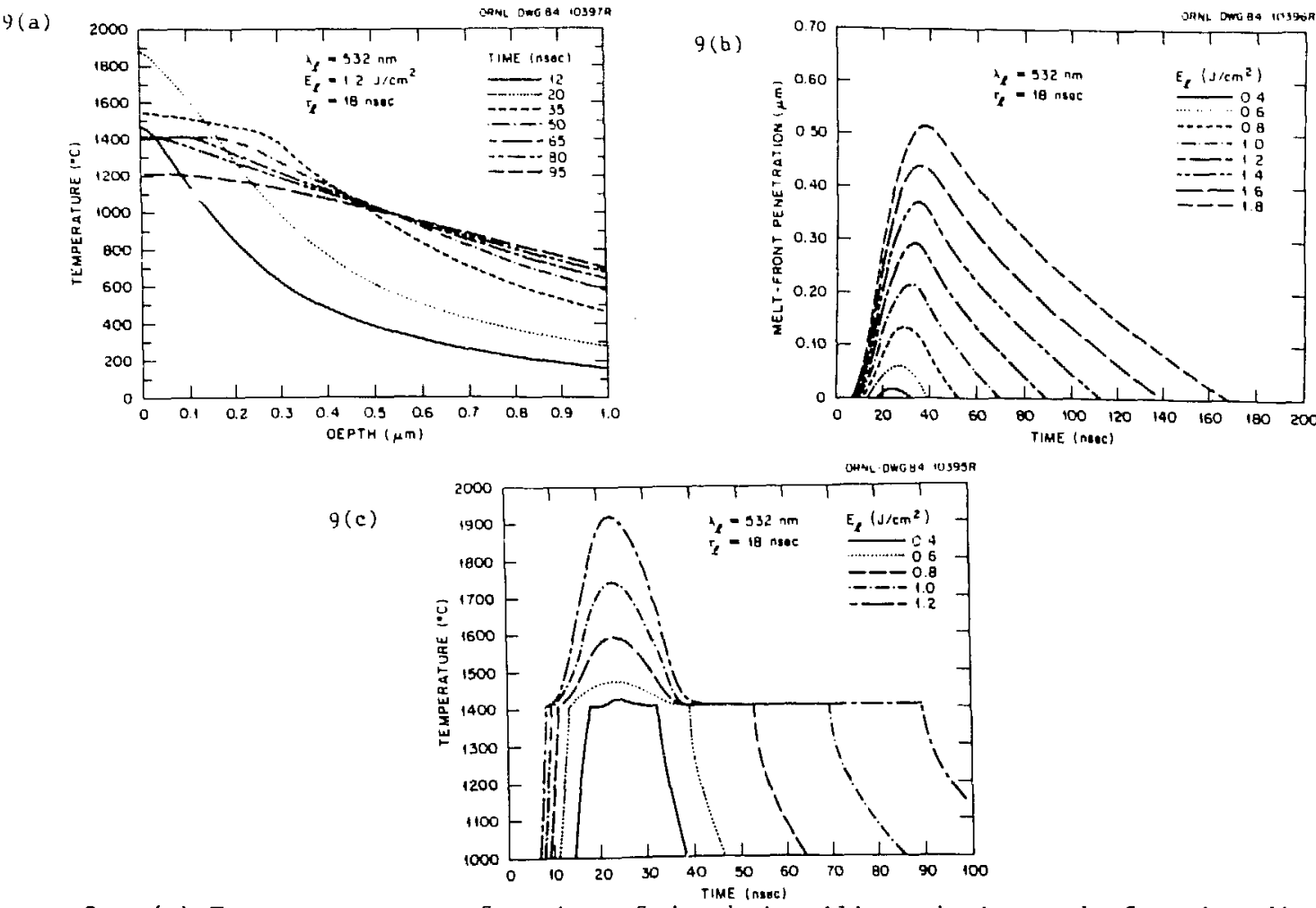

Figure 9. (a) Temperature as a function of depth in silicon during and after irradiation for several times after the beginning of the laser pulse. (b) Melt-front position as a function of time after laser irradiation for several different laser energy densities. (c) Surface temperature as a function of time after laser irradiation for several different laser energy densities. The laser pulse simulated was a frequency-doubled Nd:YAG laser (532 nm) with 18 nanosecond pulse width (Erom Chapter 4 of Ref. 1).

In addition to determining the time of the onset of melting and the melt duration from melting model calculations, a whole host of additional parameters can be calculated. In Fi.g. 9. three typical plots extracted from the melting model calcuiations are shown; these calculations were perfurmed to simulate laser irradiation of silicon with a frequencydoubled Nd:XAG laser (532 nm), with a pulse width of 18 nanoseconds full width at half maximum. A fundamental calculation is the temperature versus depth, shown in Fig. 9 (a) for a pulse energy density of $1.2 \mathrm{~J} / \mathrm{cm}^{2}$ at several times after the beginning of the laser pulse. 
The break in some of the curves at $T=1410^{\circ} \mathrm{C}$ locates the position of the liquid-solid interface, or melt front, at the time for which the curve is drawn. The profiles curves of

Fig. 9 (a) can be directly compared with the results of the time-resolved x-ray measurements. Froin the results of a series of calculations such as those represented in Fig. 9(a), the position of the melt front can be determined as a function of iime for a given pulse energy density, and plotted to yield the curves shown in Fig. 9(b). The derivative of the melt front position with respect to time gives the melt front velocity at each time. It can be seen from Fig. 9(b) that, for energy densities $20.4 \mathrm{~J} / \mathrm{cm}^{2}$, the melt front penetrates very capidly inco the solid before reaching ics maximum penetration. Near the maximum penetration, the melt-front velocity first drops sharply, then changes sign, and the melt front recedes back to the surface at several meters per second. These are the principal features of the melt-front calculations and their essential validity is confirmed by che variety of experilencs presented in the last section.

Figure ?(c) shows the surface temperature $T_{S}$ of the $S i$ as a function of the time after the beginning of pulses of several different energy densities. Ts initially increases very rapidly unti: it reaches the melting temperature at $1410^{\circ} \mathrm{C}$, where it pauses momentarily until the latert heat of melting is absorbed and then begins to rise again (if the energy density is larg، enough) to some maximum value. On cooling, the process is reversed except that $T_{S}$ drops qu: ckly to $1410^{\circ} \mathrm{C}$. Where it remains for a long period of time. Once the melt front reaches the surface of the sample, the surface remperature again drops rapidly for several nanoseconds before the rate of temperature decrease slows down. This behavior has been observed experimentally by Jellison and Lowndes using time-resolved ellipsometry.5

Recently, Wood and Geist 27 have developed the numerical techniques needed to simulate the laser irradiation of amorphous silicon. These calculations include the capability rf handling undercooling, incerface kinetics, and nucleation in the heat-flow framework. Their calculations have been very successful in reproducing the experimental features of the pulset laser irradiation of amorphous $\mathrm{Si}$ reported in Refs. 18 and 19 and discussed in the previuus section.

\section{Summary}

We have shown that the process of pulsed laser irradiation of $\mathrm{Si}$ and Ge is well understood. When a laser pulse of sufficient energy density is incident upon a crystalline $\mathrm{Si}$ or Ge surface, the surface first heats up and then melts. The melt front propagates into the material very rapidly at Eirst, slows down, pauses, and then returns to the sample surface. If the velocity of the receding melt front is slow enough, single crystalline material is regrown, otherwise, amorphous material is regrown. A host of experimental techniques have been used to study these phenomena, including time-resolved reflectivity, timeresolved transmission, time-resolved x-ray diffraction, and time-resolved electrical conductivity experiments. Melting model calculations have also been performed, showing that the results of all these experimencs can be understood in detail by invoking a concepually simple thermodynamic model of the pulsed laser irradiation process.

Throughout all these studies, excimer lasers have proven to be extremely valuable experimental tools. They are reliable, have good transverse beam homogeneity, have only small pulse-to-pulse differences in pulse shape and energy density, and their uv output is absorbed in the first 10-20 nm of most materials. However, some improvements in excimer laser rechnology would be very helpful for experimental scientists working in this area:

1) It would be very nice to have a reliable picosecond excimer laser. Such a laser would open up a whole class of experiments, including further amorphization experiments and investigations of the electron-hole plasma created by radiation above the direct band gap.

2) For the ainorphization experiments, it would be particularly useful to have an excimer laser with variable pilse width, in the range of 1 ps to $>1$ ns.

3) From the point of view of any of these experiments, it would be very helpful to know precisely the transverse homogeneity of the laser beam, at least on the 1 um scale.

\section{Acknowledgments}

Research sponsored by the Division of Materials Sciences, U.S. Department of Energy under contract No. DE-ACO5-840R21400 with Martin Marietta Energy Systems, Inc.

\section{References}

1. "Pulsed Laser Processing of Semiconductors," (Semiconductors and Semimetals, Vol. 23, ed. by R. F. Wood, C. W. White, and R. T. Young, Academic Press, 1984).

2. W. R. Sooy, M. Geller, and D. P. Bortfeld, Appl. Phys. Lett. 5, 54 (1964). 
3. D. H. Auston, C. M. Surko, T. N. C. Venkatesan, R. E. Slusher, and J. A. Golovchenko, Appl. Phys. Lett. 33, 437 (1978).

4. D. H. Lowndes, G. E. Jelilison, Jr., and R. F. Wood, Phys. Rev. B 26, 6747 (1982).

5. G. E. Jellison, Jr. and D. H. Lowndes, Appl. Phys. Lett. $41,594(1982)$.

6. G. E. Jellison, Jr., D. H. Lowndes, D. N. Mashburn, and R. F. Wood, Phys. Rev. B 34, (1986).

7. G. E. Jellison, Jr. and D. H. Lowndes, Appl. Phys. Lett. 47, 718 (1985).

8. D. A. G. Bruggeman, Ann. Phys. (Leipzig), 24,636 (1935).

9. N. Bloembergen, H. Kurz, J. M. Liu, and R. Yen, Mat. Res. Soc. Symp. Proc. 4. 3 (1982).

10. E. J. YofEa, Phys. Rev. B 21, 2415 (1980).

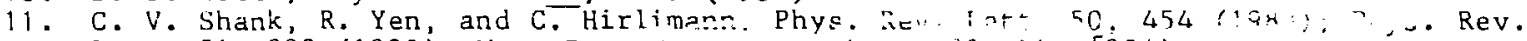
Lett. 51,900 (1983); Mat. Res. joc. Eyup. ízoc. 23, jj (1984).

12. B. C. Larson, C. W. White, T. S. Noggle, and D. Milis, Fhys. Rev. Lett. 48, 337 (1982); B. C. Larson, C. W. White, T. S. Noggle, J. F. Barhorst, and D. M. Mills, Appl. Phys. Lett. 42, 282 (1983).

13. B. C. Larson, J. Z. Tischler, and D. M. Mills, J. Mat. Res. 1, 144 (1986).

14. G. J. Galvin, M. O. Thompson, J. W. Mayer, R. B. Hammond, N. Paulter, and P. S. Peercy, Phys. Rev. Lett. $48,33(1982) ; G . J . G a l v i n$, M. O. Thompson, J. W. Mayer, P. S. Peercy, R. B. Hammond, and N. Paulter, Phys. Rev. B 27, 1079 (1963).

15. M. O. 'Thompson, J. W. Mayer, A. G. Cullis, H. C. Webber, N. G. Chew, J. M. Poate, and D. C. Jacobson, Phys. Rev. Lett. 50, 896 (1983).

16. P. L. Liu, R. Yen, N. Bloembergen, and R. T. Hodgson, Appl. Phys. Lett. 34,864 (1979): J. M. Liu, R. Yen, H. Kurz, and N. Bloembergen, Appl. Phys. Lett. 39, 755 (1981); R. Tsu, R. T. Hodgson, T. Y. Tan, and J. E. E. Baglin, Phys Rev. Lett. 42, 1356 (1979).

17. P. H. Bucksbaum and J. Bokor, Phys. Rev. Lett. 53, 182 (1984).

18. M. O. Thompson, G. J. Galvin, J. W. Mayer, P. S. Peercy, J. M. Poate, D. C. Jacobson, A. G. Cullis, and N. G. Chew, Phys. Rev. Lett. 52, 2360 (1984).

19. D. H. Lowndes, G. E. Jellison, Jr., S. J. Pennycook, S. P. Withrow, D. N. Mashburn, and R. F. Wood, Mat. Res. Soc. Symp. Proc. 51, 131 (1986); D. H. Lowndes, G. E. Jellison, Jr., S. J. Pennycook, S. P. Withrow, and D. N. Mashburn, Appl. Phys. Lett. 48, 1389 (1986).

20. R. F. Wood and G. E. Giles, Phys. Rev. B 23, 2923 (1981).

21. C. J. Glassbrenner and G. A. Slack, Phys. Rev. 134, A1058 (1964).

22. R. Hultgren, P. D. Desai, D. T. Hawkins, D. T. Geíser, K. K. Keliy, and D. D. Wayman, "Selected Values of the Thermodynamic Properties of the Elements," (American Society for Metals, Metals Park, OH, 1973).

23. G. E. Jellison, Jr. and F. A. Modine, Appl. Phys. Lett. 41, 180 (1982); G. E. Je11ison, $\mathrm{Jr}$. and H. H. Burke, J. Appl. Phys. 60,841 (1986).

24. L. Vinä, S. Logothetidis, and M. Cardona, Phys. Rev. B 30, 1979 (1984).

25. K. M. Shvarev, B. A. Baum, and P. V. Gel'd, High Temp. 15,548 (1977).

26. J. N. Hodgson, Phil. Mag. $\underline{6}, 509$ (1961). 27. R. F. Wood and G. A. Geist, Phys. Rev. B 34, (1986); Phys. Rev. Lett. 57, 873
$(1986)$. 